\title{
INTRADERMAL T.A.B. REACTIONS
}

\author{
Lieutenant-Colonel J. E. NOBLE \\ M.C.Path., M.R.C.S., L.R.C.P., D.T.M. \& H., R.A.M.C. \\ David Bruce Laboratories
}

\section{Introduction}

PROPHYLACTIC immunization with enteric (TAB) vaccine and combined enteric and tetanus (TABT) vaccine by the intradermal route is now an accepted and well tried procedure in the Services. Noble (1963) reviewed all the reactions of sufficient severity to warrant the suggestion that these vaccines may have been unduly toxic and found only 42 unacceptable reactions from approximately 7 million issued doses. He came to the conclusion that the incidence of severe reactions was negligible.

Many authors including Tuft (1931 and 1940), Valentine et al (1935), Perry (1937), Van Gelder and Fisher (1941), Naumer and Nerb (1943), Leibovitz (1943), Kamp (1943) and Luippold (1944) have advocated the intradermal route for the administration of $T A B$ vaccine and all have stressed the marked absence of both general and local reactions when this method is adopted. Recently however Bardhan, Dutta and Krishnaswami (1963) and Zuckerman (1964a and b) have suggested that unacceptable local reactions occur when TAB is administered intradermally. Field (1964) has critically appraised the article by Bardhan et al (1963) and found several discrepancies in both the vaccine and the technique used by these authors. Zuckerman (1964a and b) while not wishing to detract from the merit of intradermal TAB vaccine, drew adverse publicity to the vaccine produced by the David Bruce Laboratories for issue to the Services, and used by him in his laboratory. As a consequence it was decided to study and describe in detail the local lesion produced by the David Bruce Laboratories intradermal TAB vaccine.

\section{Materials and Method}

Twenty volunteers, from the staff of the David Bruce Laboratories were inoculated with $0.1 \mathrm{ml}$. of intradermal TAB (DBL batch No. 41) into the skin of the outer aspect of the arm behind the posterior border of the distal portion of the deltoid muscle. The response obtained was studied at $6,12,18,24,36,48$ and 72 hours after inoculation.

\section{Results}

One volunteer had a mild rigor at 12 hours, his temperature was $101^{\circ} \mathrm{F}$ and at 18 hours he had typical coryza with running nose, congestion, injected fauces, and vague muscle and joint pains: The local reaction was identical to the other 19 volunteers in the experiment. He recovered in three days without hospitalization.

The responses to the TAB inoculations are described below and all followed an identical pattern with little variation between the smallest and largest reactions.

"At 6 hours the site of skin puncture showed a $5-10 \mathrm{~mm}$. firm indurated nodule surrounded by an erythematous flare of a further $5-10 \mathrm{~mm}$. At 12 hours the central induration was less well defined, softer to palpation and varied from $20-30 \mathrm{~mm}$. in diameter. The erythematous flare extended the overall size of the lesion to between 50 and $70 \mathrm{~mm}$. (2-3 in.). In no case did the reaction increase further in size. Gradually between 12 and 24 hours the demarcation between central and peripheral parts of the lesion disappeared 
to leave an erythematous soft brawny intracutaneous oedema similar to the photograph by Zuckerman (1964b). Resolution rapidly occurred, the erythema had faded by 48 . hours and by 72 hours only a small intradermal nodule of between 5 and $8 \mathrm{~mm}$. in diameter remained.

No pyrexia occurred except in the volunteer described above with concomitant coryza. Two volunteers complained of slight axillary tenderness but no glands were palpable and deep axillary palpation was possible without increase of the soreness. Not one volunteer complained of pain. Leading questions as to the presence of pain led to the typical response, "I am conscious of the presence of the reaction but it does not hurt". Tenderness to touch was minimal in all cases. There was no loss of function throughout. One volunteer played golf, two played soccer and one a game of rugby football at about 18 hours after inoculation with no evidence of adverse effects due to the injections.

\section{Discussion}

The reaction to subcutaneous $\mathrm{TAB}$ inoculations consisting in many cases of a severe local lesion extending from shoulder to elbow and a general reaction of rigors and pyrexia often requiring two days bed rest, is in danger of being forgotten by many vaccinators. The adjectives negligible and acceptable, frequently used to describe the response to intradermal inoculation require clarifying in the light of some current adverse criticism of this procedure (Zuckerman 1964a and b).

Taking into consideration the reactions described above and which conform very accurately to the so called acceptable reaction normally expected by the writer, they are in no way negligible except by comparison to the profound reactions often encountered when TAB vaccine was administered by the subcutaneous route. It is recommended that the word " negligible" be discontinued in this context. Furthermore it is contended that "acceptable" means a reaction which causes no general response, no undue pain or adenitis and no loss of function whatsoever. It is not intended to imply the absence of what might be described as an unsightly red wheal of temporary duration lasting approximately 48-72 hours. Hooper (1964) and MacFarlane (1964) appear to support this view. The 42 severe reactions in 7 million issue doses described by Noble (1963) were unacceptable by these standards.

Only about six complaints of undue toxicity of the David Bruce Laboratories TABT and $T A B$ vaccines are received in a year. Investigation of these complaints is often revealing and most are invalid. The three most common causes of reaction are in our opinion:- intradermal inoculation into the flexor surface of the forearm in direct contravention of the instructions both issued with every bottle of vaccine and in the Memorandum on Immunological Procedures (1961); inoculation into the correct site but at some time during a developing smallpox vaccination with a resulting additative reaction for which the TAB is held responsible; and the inoculation of subjects of known hypersensitivity to $\mathrm{TAB}$ or with a known allergic diathesis in some form or another. The few severe reactions which cannot be accounted for by the foregoing reasons may often be due to faulty technique, the most frequent being subcutaneous leakage.

\section{Summary}

In view of some recent adverse criticism the normal response to David Bruce Laboratories issue TAB vaccine has been described in detail. 
It is suggested that the reaction is not negligible and this term should be discontinued in this context.

The term " acceptable reaction" has been defined.

Some common causes of unacceptable reactions are listed.

\section{REFERENCES}

Bardhan, P. N., Dutta, H. N. \& Krishmaswami, P. (1963). J. Hyg., Camb., 61, 365.

FIELD, T. E. (1964). J. roy. Army. med. Cps., 110, 110.

HOOPER, W. L. (1964). Brit. med. J., 2; 571.

KAMP, M. (1943). J. Indiana. med. Assoc., 36, 584.

LeIbovitz, A. (1943). Yale J. Biol. and Med., 15, 609.

Luippold, G. F. (1944). Amer. J. Pub. Hith., 34, 1151

MAcFarlane, L. R. S. (1964). Brit. med. J., 2, 445.

Memorandum on Immunological Procedures (1961). H.M.S.O. Code No. 13161.

Naumer, H. A. \& Nerb, L. (1943). Arch. Ped., 60, 63.

Noble, J. E. (1963). J. roy. Army Med. Cps., 109, 178.

Perry, R. M. (1937). Amer. J. Hyg., 26, 388.

TUFT, L. (1931). J. Lab. and Clin. Med., 16, 552.

TuFT, L. (1940). Amer. J. med. Sci., 199, 84.

Valentine, E., Park, W. H., Falk, K. G. \& McGuire, G. (1935). Amer. J. Hyg., 22, 44.

VAN Gel Der, D. W. \& Fisher, S. (1941). Amer. J. Dis. Child., 62, 933.

ZUCKERMAN, A. J. (1964a). Brit. med. J., 2, 119.

ZuCKERMAN, A. J. (1964b). Brit. med. J., 2, 689.

\section{'THE AUTO-CAR AS AN AMBULANCE'}

In the opinion of Regimental Surgeon Prevost (Archives Medicales Belges) the ambulance of the future will be an auto-car. Over ordinary horse-drawn wagons these cars possess many and great advantages. In the first place, the length of a sick convoy will be diminished by a third, if not a half, and it will travel at a considerably greater speed. Secondly, the occupants of an auto-car ambulance will be spared the jolting and shaking inseparable from horse-or mule-drawn vehicles when starting or halting, as well as those due to restive animals. Thirdly, the provision and carriage of bulky forage will be dispensed with. Against the auto-car can only be urged its cost and liability to go out of order. In a short time both these defects will, it is confidently anticipated, be reduced to a minimum. Another advantage in addition to those enumerated above would consist in the greater cleanliness of the auto-car. The dejections of horses and other animals are a fruitful source of complaint if not of disease. It has been calculated by Surgeon Prevost that if auto-car ambulances were to be used exclusively for military hospitals it would be possible to economise 193 men per army corps.

The Lancet, February 20, 1904, 